\title{
Genetic analysis of Rhaponticum carthamoides (Asteraceae) during restoration of natural populations in the Altai Republic
}

\author{
Irina Kuban ${ }^{1}$, Elena Zhmud $^{1 *}$, Altynai Achimova ${ }^{2}$, Natasha Williams ${ }^{3}$, and Olga Dorogina ${ }^{1}$ \\ ${ }^{1}$ Central Siberian Botanical Garden SB RAS 630090 Novosibirsk, Russia \\ ${ }^{2}$ Gorno-Altai Botanical Garden, 649218 Kamlak, Altai Republic, Russia \\ ${ }^{3}$ Colorado State University, 80523, Fort Collins, Colorado, USA
}

\begin{abstract}
Rhaponticum carthamoides (Willd.) Iljin is a rare species for Altai Republic. It possesses medicinal and fodder properties and therefore is considered a valuable resource species. Populations of this species are regularly exposed to anthropogenic impact. The impact factors are harvesting of rhizomes and cattle grazing. We have carried out the restoration of two populations of this species. The seeds for restorative planting were collected from healthy plants in the same populations, at a distance of $10 \mathrm{~km}$ or less from the restoration site. The genetic analysis of DNA fragments showed a reliable similarity of genetic diversity in planted and naturally growing individuals in each of the studied populations. Comparison showed that plants in more remote locations, which are less accessible for tourism and economic activities, were characterized by higher genetic diversity.
\end{abstract}

\section{Introduction}

In the Altai Republic, since the beginning of the 21 st century, the system of communications is developing rapidly, driven by the expansion of agriculture and tourism. The flip side of the positive economic and social development is the reduction in plant biodiversity due to overuse of natural plant resources. Such tendency can lead to the disruption of populations of the rare and especially of the valuable resource species.

Rhaponticum carthamoides (Willd.) Iljin is a valuable resource species that has been given a conservation status on the territory of the Altai Republic (AR) [1]. According to our data, the state of this species in natural populations is rapidly declining due to anthropogenic impact (excessive grazing of farm animals and harvesting of rhizomes for medicinal purposes) [2]. Consequently, natural populations of this species are in need of restoration. This is especially relevant to the populations in the Altai Republic at the Seminsky Pass (Ongudaysky District) and at the foot of Krasnaya Mountain (UstKoksinsky District, circa Kaitanak village), which are subject to substantial agricultural and tourism impact. Therefore, populations at these locations were seeded with $R$. carthamoides in an attempt to restore the diminishing natural abundance of the species.

\footnotetext{
*Corresponding author: elenazhmu@ngs.ru
} 
Our study is based on the established data of the genetic similarity between the plants from native populations and plants used for the restoration of disrupted populations. The previous research, based on the electrophoretic spectra of storage proteins, showed that, in the same locations as described above, plants of Hedysarum theinum Krasnob., introduced for restoration were identical to the native plants. Similar data is lacking for populations of $R$. carthamoides, as confirmed by our search of published sources. Therefore, the purpose of this study was a comparative analysis of the genetic similarity between the DNA spectra of the native plants and those seeded for restoration in the natural populations of $R$. in the AR.

\section{Materials and method}

Population I (P. I) of $R$. carthamoides is located on the Seminsky pass (Ongudaysky district $51^{\circ} 03^{\prime} 93^{\prime \prime} \mathrm{N}$; $85^{\circ} 39^{\prime} 43^{\prime \prime} \mathrm{E}$; altitude $1816 \mathrm{~m}$ above sea level; the number of individuals about 1200 specimens). Population II (P. II) is located near the foot of Krasnaya Mountain (Ust-Koksinsky District, in the vicinity of Kaitanak; 5005'12" N; 85²'52" E; $2010 \mathrm{~m}$ above sea level; the number of individuals - about 600 specimens). Seeds of R. carthamoides were collected in 2009, from intact native plants, at a distance of $10 \mathrm{~km}$ or less from the locations of the above described sites. These seeds were seeded in 2010, in the populations described above, and left for growing alongside the native specimens. In July 2020 , the seeded individuals had reached the age of 10 years and were in pre-generative period of development. At this time, naturally growing representatives of the species (native) and the plants seeded for restoration (planted) were collected for our study.

DNA was isolated from the fragments of the leaf blades of $R$. carthamoides, which were dried in the laboratory conditions. In Population I, nine native and nine planted representatives were studied for comparison on two square plots (s.). Each square plot comprised an area of one square meter. On square \#1, five individuals (No. 1.1 - 1.5), and on square \#2, four individuals (No. 2.1-2.4) were sampled. In Population II, six native and fourteen planted representatives were studied on three squares: on square \#6 - six samples (No. 6.1-6.6); on square \#7 - five samples (No. 7.1-7.5); on square \#8 - three samples (No. 8.1-8.3). DNA was isolated using standard method with some modifications. For comparison, primers previously tested for this species were used [4]. The DNA concentration was, on average, $71.9 \mathrm{ng} / \mu \mathrm{L}$ (13.3-261). The purity of the obtained DNA samples was about $1.11(0.26-1.97)$. data was obtained by analyzing the mean values of the spectra difference coefficients using nonparametric test (Mann-Whitni U-Test; StatSoft Statistica 6.0).

\section{Results and discussion}

The distance between the studied populations I and II of $R$. carthamoides is more than 300 $\mathrm{km}$. According to the Kuban et al. study [5], individuals in these two populations are capable of self-maintenance under a moderate anthropogenic impact [5]. These sites differ in their accessibility for tourism and economic activity. The Seminsky pass (P. I) is located next to a large settlement (the regional centers of Shebalino and Onguday) and next to the main transport route of the Altai Republic - the Chuisky tract. In summer, these sites are subject to cattle grazing. As a result, more than $50 \%$ of $R$. carthamoides specimens lacked inflorescences in 2020. Also, an additional impact may come from a hiking trail that runs alongside this populations. Population 2 is located in a remote and quite inaccessible area, in the vicinity of a small settlement, far from the Chuisky tract and other main transportation routes. During warm seasons, this area is sometimes visited by small 
excursion groups from neighboring tourist centers; also, the roots of $R$. carthamoides are harvested by local residents. Therefore, anthropogenic load seems a lot greater in the locale of P. I than that of P. II.

Analysis of the variability of the distribution of DNA fragments showed high similarity of the spectra in individuals within each of the populations (Table 1). In addition, the difference in all comparison variants between the native and planted individuals was specific to each plot.

Table 1. Average values of the differentiation coefficients of DNA spectra in individuals of Rhaponticum carthamoides in the Altai Republic (2020)

\begin{tabular}{|c|c|c|c|c|c|}
\hline Site No & Comparison options & $\mathrm{M} \pm \mathrm{m}$ & $\mathrm{Cv}, \%$ & $\min$ & $\max$ \\
\hline \multirow{4}{*}{ I } & $\mathrm{N}^{*}$ & $11.5 \pm 1.3$ & 33.7 & 7.6 & 18.6 \\
\cline { 2 - 6 } & $\mathrm{N} \_1$ & $11.2 \pm 1.3$ & 33.4 & 4.3 & 15.7 \\
\cline { 2 - 6 } & $\mathrm{N} \_2$ & $7.2 \pm 1.0$ & 42.1 & 1.3 & 11.3 \\
\cline { 2 - 6 } & square 1 & $12.5 \pm 1.7$ & 29.9 & 8.5 & 17.9 \\
\hline \multirow{4}{*}{ II } & square 2 & $5.4 \pm 1.1$ & 39.9 & 3.5 & 7.2 \\
\cline { 2 - 6 } & N* & $28.7 \pm 1.7$ & 14.8 & 24.3 & 35.0 \\
\cline { 2 - 6 } & N_6 & $27.1 \pm 2$ & 18.2 & 16.2 & 26.9 \\
\cline { 2 - 6 } & N_8 & $37.1 \pm 2.0$ & 13.4 & 19.8 & 37.5 \\
\cline { 2 - 6 } & square 6 & $19.9 \pm 1.5$ & 19.1 & 16.2 & 26.9 \\
\cline { 2 - 6 } & square 7 & $28.0 \pm 3.7$ & 29.3 & 19.8 & 37.5 \\
\hline
\end{tabular}

Designations: *N - native samples; $N \_1 \ldots N \_8-N \_1 \ldots N \_8-$ comparison of native and planted samples; No 1-2, 6-8 - average values of the differentiation coefficients of the DNA spectra of the individuals planted in the population.

Our research showed low differences in DNA spectra of individuals from P. I. According to the spectra of DNA fragments, the native and planted individuals of $R$. carthamoides were similar. DNA spectra in more than $88 \%$ of native plants in this population are homogeneous (Table 1). On the experimental sites, $87-95 \%$ of the planted individuals also showed such similarity. Thus, both studied groups of individuals in P. I (native and planted) were characterized by low and comparable levels of differences in the spectra of DNA fragments.

In contrast, the native individuals in P. II exhibited much higher genetic diversity than those in P. I. This was shown by the higher average coefficients of difference in the distribution of DNA fragments. That is, native individuals from P. II were more heterogeneous than those in P. I. The spectra of their DNA fragments were similar, on average, in about $70 \%$ of the individuals (Table 1). Between the native and planted representatives of $R$. carthamoides in this area, the degree of genetic homogeneity was comparable, and comprised about $72-80 \%$ (Table 1). 
Thus, the genetic heterogeneity of individuals, according to the spectra of DNA fragments, was higher in a relatively more remote and inaccessible habitat of P. II. This data was obtained by analyzing the mean values of the spectra difference coefficients using nonparametric test (Mann-Whitni U-Test; p-level $=0.0014<0.05$ ).

Samples of $R$. carthamoides from two different habitats turned out to be closely related, but at the same time reliably genetically differentiated. According to the cluster analysis, the samples were divided into 2 main groups. About $30 \%$ of individuals in each cluster were characterized by the distribution of individual DNA components similar to the samples from another cluster. Thus, 20 sample plants were assigned to the first cluster, most of which (14 individuals or 70\%) grew in P. I, and the remaining 6 - in P. II (Fig. 1). Accordingly, 18 sample plants were assigned to the second cluster, out of which 14 individuals grew in P. II (77.8\%), and the rest - in P. I. Each cluster included both native and planted individuals, which confirms high degree of similarity of the spectra of DNA fragments in the plants of both studied areas.

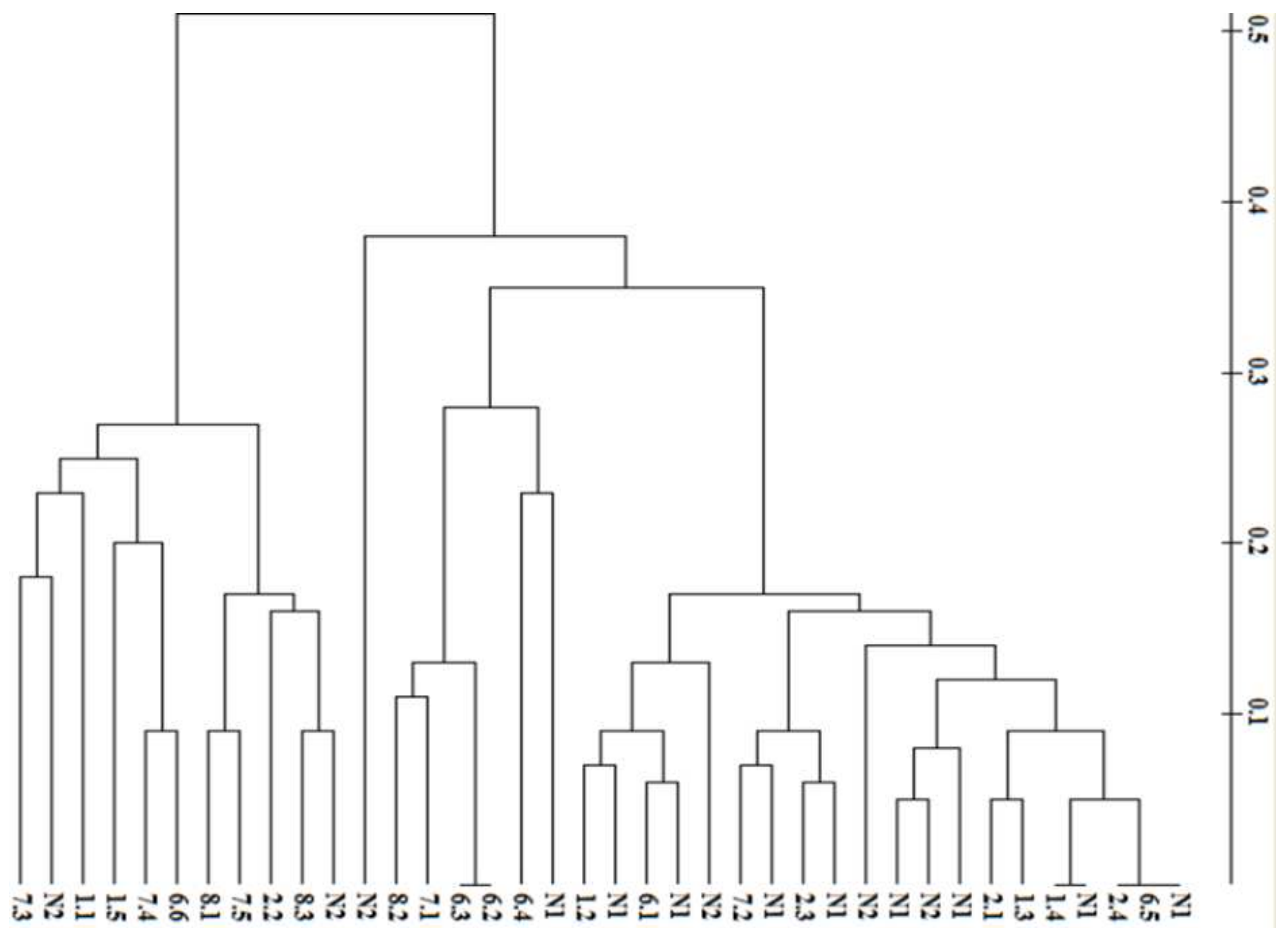

Fig. 1. Dendrogram of the differences in the spectra of DNA fragments in individuals of Rhaponticum carthamoides in natural populations of the Altai Republic. Designations: N1 - P. I (Seminsky pass); N2 - P. II (foot of Krasnaya Mountain). N - native individuals; 1.2 - 8.3 - planted individuals of natural origin. The scale at the top is genetic distance (Nei, 1979).

\section{Conclusion}

Thus, the genetic analysis confirmed high degree of similarity between native plants of $R$. carthamoides and individuals planted for restoration in the studied populations. High degree of genetic similarity in the identified DNA fragments suggests that seeds collected from indigenous populations of Rhaponticum carthamoides within the distance of up to 10 $\mathrm{km}$ from the locale of the restoration site can be successfully used to restore the degraded populations of the species. 
This work was supported by the regional project of the Russian Foundation for Basic Research and the Government of the Altai Republic No. AAAA-A21-121011290025-2 and the basic project of the CSBG SB RAS № AAAA-A21-121011290025-2

\section{References}

1. Red Book of the Altai Republic (plants) (Gorno-Altajsk, 2017)

2. N. A. Nekratova, A. V. Kurovskiy, M. N. Shurupova, Ukrainian Journal of Ecology 10(4) (2020)

3. O. Yu. Vasilyeva, O. V. Dorogina, I. N. Kuban, I. Ya. Sarlaeva, L. V. Buglova, Horticulture and viticulture 4(214) (2018)

4. I. N. Kuban, O. V. Dorogina, E. V. Zhmud, Plant Life of Asian Russia, 3(31) (2018) 